\title{
Trypophobia: Heart rate, heart rate variability and cortical haemodynamic response
}

An Le, Geoff Cole, Arnold Wilkins ${ }^{1}$

University of Essex, Colchester, CO4 3SQ

Keywords: Cardiovascular Reactivity, Haemodynamic Response, Phobia, Visual Discomfort

1.Address for correspondence

Prof A Wilkins

Department of Psychology

University of Essex

Colchester

$\mathrm{CO} 43 \mathrm{SQ}$

Tel +44 1223812537

Email: arnold@essex.ac.uk 


\begin{abstract}
Background: Trypophobia is a common condition in which sufferers are averse to images of small holes arranged in clusters. Methods: We used photo-plethysmography to examine cardiovascular correlates and near infrared spectroscopy to examine cortical correlates of the phenomenon in order to validate the Trypophobia Questionnaire and explore the several interlinked explanations of the disorder. Results: Trypophobic images were found to increase heart rate and heart rate variability, but only in individuals with high scores on the Trypophobia Questionnaire. Trypophobic images were also found to elicit larger haemodynamic responses in posterior cortical areas, but again only in individuals with high scores. Limitations: The results are consistent with a contribution from both parasympathetic and sympathetic systems. Conclusion: The data demonstrate the validity of the Trypophobia Questionnaire and show an involvement not only of the autonomic system but cortical mechanisms including cortical hyperexcitability.
\end{abstract}

Keywords: Cardiovascular Reactivity, Haemodynamic Response, Phobia, Visual Discomfort

\title{
1. Introduction
}

Trypophobia is a condition in which symptoms such as itchiness and heart palpitations are induced by a wide variety of images that contain clusters of small objects, usually holes (Cole \& Wilkins, 2013). The condition is associated with clinically significant morbidity and comorbidity (Vlok-Barnard \& Stein, 2017). Inducing stimuli can be natural (e.g., honeycomb) or human-made (e.g., aerated chocolate), and are thought to induce symptoms that fall into three categories: (1) skin-related; (2) cognitive or (3) physiological (Le, Cole, \& Wilkins, 2015). Although a number of internet-based support groups currently exist in which sufferers relate how debilitating the condition can be, trypophobia, together with the majority of other phobias, is not specifically mentioned in the DSM- 5 (American Psychiatric Association, 2013). One of the curious aspects of trypophobia is that the visual stimuli that induce the condition are seemingly innocuous and carry no semantic properties associated with threat (Cole \& Wilkins, 2013). Indeed, one of the most commonly cited trypophobic stimuli is a harmless plant: the seed head of the lotus flower, Figure 1.

\section{INSERT FIGURE 1 ABOUT HERE}

Studies of the physiological correlates of trypophobia have involved measurements of pupil size, skin resistance, heart rate, and evoked potentials. During observation of trypophobic images pupils have been shown to constrict, consistent with a parasympathetic response (Azenburg, Hickey, \& Lourenco, 2017). On the other hand, Pipitone and colleagues (2017) found electrodermal activity suggestive of a sympathetic response but 
failed to show an effect on heart rate, as might have been expected were the sympathetic system involved. Two ERP studies have shown enhanced posterior negativity in trypophobia consistent with enhanced autonomic processing of emotionally significant visual information (Van Strien and Van der Peijl, 2018; Wanegger, Schwab \& Schienle, 2019), Whereas Van Strien and Van der Peijl showed enhanced early posterior negativity, Wanegger et al. showed enhanced late negativity. It is however unclear whether these responses reflect enhanced attention or enhanced emotion or both.

To complement these studies we investigated heart rate and heart rate variability, which are sympathetic responses known to reflect anxiety. We also measured the cortical haemodynamic response, known to be greater in response to uncomfortable visual stimuli (Le et al., 2017). Although it has previously been shown that trypophobia is not greater in anxious individuals, i.e., it is not a reflection of trait anxiety (Pipitone et al. 2017; Le et al., 2018), it is possible that trypophobic visual stimuli induce anxiety as a state response. Indeed, some of the questions in the Trypophobia Questionaire (TQ; Le et al., 2015) of are designed to index symptoms that would typically reflect anxiety. Cardiovascular reactivity (i.e., heart rate and its variability) may be expected to be affected by the participants' experience of disgust, according to the review by Kreibig (2010). For example, it was reported that contamination-related disgust was distinguished from other negative emotions in so far as the former increased heart rate variability. Thus, the Autonomic Nervous System (ANS) activity produced by emotions can distinguish between positive and negative emotions, and also between negative emotions (Ekman, Levenson, \& Friesen, 1983).

A large haemodynamic response is a reliable marker of discomfort and visual stress. For instance, recent neuroimaging studies in healthy individuals and individuals who suffer migraine have been consistent in demonstrating that those who experience visual discomfort or photophobia exhibit an unusually large haemodynamic response to stressful visual stimuli (Wilkins, 2015). If trypophobia can also be characterized as a visual stress response, we should observe increased haemodynamic activity in visual areas of the cortex in trypophobic individuals when viewing trypophobic stimuli; an effect that should not occur in non- trypophobic individuals.

In summary, we examined how the physiological responses to trypophobic images are influenced by the emotions of disgust/fear as opposed to the more direct contributions from visual stress.

\subsection{Method}

\subsubsection{Participants}

Two-hundred and fourteen students from the University of Essex were surveyed using Qualtrics (Qualtrics Labs Inc, 2013) from which a convenience sample was selected. The sample was divided into two groups on the basis of their score on the TQ (Le et al. 2015). Fourteen participants ( 1 male, 13 female) with TQ scores above 31 (range: $32-48$, mean 38.6 , SD 5.8), aged $18-26$, and 28 participants (8 male, 19 female) who scored less than 31 (range: $17-31$, mean 22.0, SD 4.9), aged 18-39. According to Le et al., a TQ score above 31 is a suitable criterion for trypophobia. The University of Essex Research Ethics Committee granted approval (approval code AL1402). 


\subsubsection{Procedure}

Ten random trypop 5.8hobic and ten neutral images from Le et al. (2015) were used. The images were shown to the participant using a PowerPoint slideshow with timing parameters similar to those used in a study by Haigh et al. (2103). The duration for each stimulus was $16 \mathrm{~s}$, and these were separated by a grey slide having a random duration of between 27 and 36 seconds. A white/black square (approximately $30 \times 30 \mathrm{~mm}$ ) was placed on the lower right corner for each stimulus/grey slide to trigger a photo diode used to indicate stimulus onset.

The slideshow was presented on an UltraSharp 2408WFP Widescreen 24 inch LCD

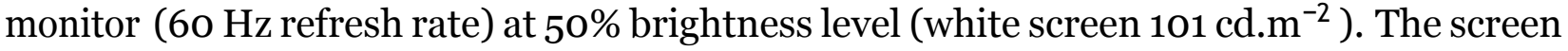
resolution was $1920 \mathrm{x}$ 1200. Each participant was seated approximately $0.7 \mathrm{~m}$ from the display, at which distance the height of the image subtended 24 degrees of visual angle. Participants were asked to watch the slideshow, while keeping movement to a minimum.

A NeXus 10 device (Mind Media BV, The Netherlands) and an associated Blood Volume Pulse (BVP) sensor were used for photoplethysmography (PPG). The BVP sensor (sampled at $128 \mathrm{~Hz}$ ) was secured on the middle finger of the non-dominant hand, which rested on the participant's lap.

\subsubsection{Data analysis}

To obtain clear peaks and thereby facilitate analysis, the PPG signal was converted into the acceleration plethysmogram (APG), which is the second derivative of the PPG signal (Elgendi, Jonkman, \& DeBoer, 2011). The peaks of the APG signal (the largest positive value) represented the onset of each heartbeat. A moving average filter across eleven data points (Lao et al., 2012) was applied to the APG signal in order to remove high-frequency noise. HR was obtained by counting the number of peaks during stimulus presentation. The number of peaks (i.e., beats) during the stimulus was expressed as beats per minute (BPM).

The root mean square of the successive differences (RMSSD; Cacioppo, Tassinary, \& Berntson, 2007) was obtained as a measurement of heart rate variability (HRV). It uses the successive differences between heart beats, and has been recommended as an estimate of the short-term components of HRV (Task Force, 1996). For each participant, the average BPM (HR) and RMSSD (HRV) were obtained for the two image categories (trypophobic and neutral).

An 8-channel split-receiver NIRS system (MK II; Artinis Medical Systems BV, Zetten, The Netherlands) was used to measure the haemodynamic responses, sampled at a rate of $10 \mathrm{~Hz}$. The posterior channels were based on two receivers and six transmitters. The receivers were placed as described by Haigh et al. (2013), i.e., 30mm from either side of the midline and $20 \mathrm{~mm}$ above the inion. For the left hemisphere, three transmitters were placed $35 \mathrm{~mm}$ from the receiver, at $\mathrm{O}^{\circ}, 45^{\circ}$, and $90^{\circ}$ relative to a vertical midline. A symmetrical placement was applied for the right hemisphere. The optode placement for the frontal channels included two receivers and two transmitters. The receivers covered positions $\mathrm{FP} 1$ and $\mathrm{FP} 2$ of the 10-20 system of electrode placement. Both transmitters were placed $35 \mathrm{~mm}$ above the optode of the respective receiver.

To obtain the required optical density, the path length factors (DPF) of 7.25 (central 
forehead) and 8.75 (occipital areas; $10 \mathrm{~mm}$ above the inion) were used (Zhao et al., 2002). The raw signal for each channel was filtered with a running median of 31 samples to remove cardiac artifacts (Haigh et al., 2013). To remove any systematic drift in the signal, the detrend function in MATLAB(C) was applied to the filtered data. The oxyhaemoglobin and deoxyhaemoglobin measurements correlated negatively and strongly $(\mathrm{r}(34)=-.75, \mathrm{p}<$.001). Thus, only the oxyhaemoglobin responses, the larger signals, were used in the subsequent analyses.

The baseline was calculated from the response 10 s before stimulus onset for a period of $10 \mathrm{~s}$, and the stimulus response was calculated $6 \mathrm{~s}$ from the stimulus onset for a period of 10s. This was to allow the signal to reach its maximum (Haigh et al., 2013). An overall signal amplitude was obtained by subtracting the baseline response from the signal during the last 10 s of the stimulus. In order to differentiate between responding and nonresponding channels, the signal amplitude for the stimulus was compared to the variation in the baseline. If the ratio of the signal amplitude to the standard deviation of the baseline was less than 0.5, then the channel was considered as unvarying and rejected from further analysis.

\section{Results}

\subsection{Cardiovascular reactivity}

One participant was not comfortable wearing the sensor and PPG data for this participant were not obtained. Forty-one participants were therefore included in the subsequent analysis. The mean heart rate (HR) in beats per minute (BPM) and heart rate variability (root mean square of the successive differences; RMSSD in ms) for each image category, separated by group, are summarized in Table 1 . There was no consistent change through the course of stimulus presentation, and no suggestion of a biphasic heart rate profile.

Table 1. Mean (SD) BPM and root mean square of the successive differences RMSSD (ms) for trypophobic and neutral images, separated by group (control or trypophobic).

\begin{tabular}{clll}
\hline & Image category & $\begin{array}{l}\text { Control group } \\
(N=27)\end{array}$ & $\begin{array}{l}\text { Trypophobic group } \\
(N=14)\end{array}$ \\
\hline BPM & Trypophobic images & $73.7(11.8)$ & $78.1(14.2)$ \\
& Neutral images & $73.7(12.2)$ & $75.9(13.4)$ \\
RMSSD & Trypophobic images & $51.9(23.9)$ & $63.7(43.3)$ \\
$(\mathrm{ms})$ & Neutral images & $53.0(25.0)$ & $56.7(32.2)$ \\
\hline
\end{tabular}

3.1.1 Heart rate. A 2 (image category) $\mathrm{x} 2$ (group) mixed ANOVA with repeated-measures on the first factor was conducted in order investigate the effect of image type and condition on HR. A significant interaction was found, $\mathrm{F}(1,39)=8.84, \mathrm{p}<.01, \eta 2=.16$. A significant main effect was also found for image type, $\mathrm{F}(1,39)=8.00, \mathrm{p}<.01, \eta 2=.14$, but not for group, $\mathrm{F}<1$. The significant interaction was investigated further by evaluating the simple main effects of images separately for trypophobic and control groups. The results revealed that trypophobic images were associated with a higher HR (relative to neutral images) among the trypophobic group, $\mathrm{F}(1,39)=12.8, \mathrm{p}<.001, \eta 2=.25$, but not in the control group, $\mathrm{F}<1$. 
3.1.2. Heart rate variability. Similarly to the above, a 2 (image category) $\mathrm{x} 2$ (group) mixed ANOVA with repeated-measures on the first factor was conducted in order investigate the effect of image type and condition on HRV. There was a significant interaction, $\mathrm{F}(1,39)=6.52, \mathrm{p}<.05, \eta_{2}=.13$, but no significant main effects for image type or group, Fs $<1$. The significant cross-over interaction was investigated further by evaluating the simple main effects of images separately for the control and trypophobic groups. The results revealed that trypophobic images were associated with a higher HRV (relative to neutral images) for the trypophobic group, $\mathrm{F}(1,39)=7.37, \mathrm{p}<.01, \eta_{2}=.16$, but not for the control group, $\mathrm{F}<1$. Overall, when viewing trypophobic images, only individuals with trypophobia had a significantly higher HR and HRV whereas control individuals did not.

\subsection{Haemodynamic response}

First, a one-way repeated-measures ANOVA was conducted to compare the effect of optode positions on the magnitude of the oxyhaemoglobin responses for the participants who had both frontal and posterior channels that met the criteria for varying channels $(\mathrm{N}$ $=10$ ). The results suggested that the magnitude of the oxyhaemoglobin responses was significantly higher for posterior channels $(\mathrm{M}=0.21, \mathrm{SD}=0.09)$ compared to anterior channels $(\mathrm{M}=0.05, \mathrm{SD}=0.07), \mathrm{F}(1,9)=40.7, \mathrm{p}<.001, \eta 2=.82$, thereby demonstrating that the cortical response to visual stimuli was larger in the occipital areas and was not simply systemic. The response in anterior channels was low and many channels were unresponsive, precluding analysis.

Based on the above results, the following analysis was conducted using only the posterior channels, in which 38 participants obtained at least one responsive channel and were accepted for further analysis. The average number of responding channels was 4.6 (out of 6). The mean (SD) amplitudes are summarised in Table 2.

A 2 (image category) x 2 (group) mixed ANOVA with repeated measures in the first factor was conducted to investigate the effect of image type and group on the oxyhaemoglobin response in the occipital areas. A significant interaction was found, $\mathrm{F}(1$, $36)=4.12, p<.05, \eta 2=0.10$. No significant main effects were found for image type or group, Fs $<1$. The significant interaction was investigated further by evaluating the simple main effects of images separately for the trypophobic and control groups. Relative to neutral images, the trypophobic images induced a larger oxyhaemoglobin response in the trypophobic group, $\mathrm{F}(1,36)=5.11, \mathrm{p}<.05, \mathrm{\eta} 2=.13$, but not in the control group, $\mathrm{F}<1$.

Table 2. Mean (SD) amplitude ( $\mu$ molar) for trypophobic and neutral images, separated by group (control or trypophobic).

\begin{tabular}{lll}
\hline & $\begin{array}{l}\text { Control group } \\
(N=26)\end{array}$ & $\begin{array}{l}\text { Trypophobic group } \\
(N=12)\end{array}$ \\
\hline Trypophobic images & $0.17(0.15)$ & $0.30(0.28)$ \\
Neutral images & $0.18(0.12)$ & $0.16(0.14)$ \\
\hline
\end{tabular}

Across participants for the neutral images there was a correlation between the amplitude of the haemodynamic response and heart rate of -0.218 and RMSSD of 0.332. For the trypophobic images the correlations were 0.307 and -0.255 . 


\section{Discussion}

Trypophobia is the condition in which individuals are averse to images of small holes arranged in a cluster (Cole \& Wilkins, 2013). The images of holes clearly increased both the heart rate and the heart rate variability relative to neutral images, but did so only for the group with high scores on the TQ. There was no difference in response to neural images between groups. These findings are consistent with a selective emotional response to trypophobic images as suggested by Imaizumi et al. (2016), and confirm the validity of the TQ. The fact that the physiological measures were closely similar for both groups when neutral images were observed is consistent with Le et al.'s (2015) finding that individuals with trypophobia are not generally more anxious.

The selective increase in heart rate and heart rate variability in individuals with trypophobia is consistent with a sympathetic response of fear. However, as noted in the Introduction, Ayzenberg et al. (2018) reported a pupillary constriction response indicative of disgust via the parasympathetic system. The disgust response hypothesis supports the work of Kupfer and Le (2017). They noted that many infectious diseases, such as smallpox and rubella, result in trypophobia-like clusters of circular shapes occurring on the skin. The authors also found a greater associated between trypophobia and feelings relating to disgust compared with feelings relating to fear. Given these findings and those of Ayzenberg et al., together with the present results, it would appear that trypophobic images induce disgust and fear, possibly a reflection of both parasympathetic and sympathetic activity. Note that even in arachnophobia, images of spiders evoke both disgust and fear (Gerdes, Uhi \& Alpers, 2008).

The amplitude of the haemodynamic response to trypophobic stimuli was clearly larger in the individuals with high scores on the TQ both when compared with neutral stimuli and with respect to individuals with low scores on the questionnaire. The haemodynamic response occurred mainly in posterior cortical areas, and was not therefore simply a reflection of a general systemic response from emotion. These data are consistent with the notion that trypophobia is due in part to a hyperexcitability of the visual cortex.

There was no consistent relationship between the size of the haemodynamic response and heart rate or heart rate variability, supporting the idea that the emotional aspects of trypophobia and the effect of the image statistics on cortical haemodynamic are largely independent.

We suggest that the condition of trypophobia is primarily due to the hyperexcitability that occurs in visual areas of the cortex in response to trypophobic stimuli in trypophobic individuals. According to this explanation, the phenomenon is akin to visual stress. Hibbard and O'Hare (2017) and Penacchio et al. (2015) have shown that visual stimuli that are uncomfortable to view and have an excess of contrast energy at mid spatial frequencies reduce the sparseness of coding in computational models of the visual cortex. Penacchio et al. (2019) also showed that reducing the levels of inhibition within the artificial neural network exacerbates the effect. Cole and Wilkins (2013) argued that any stimulus that just happens to share the central spectral property possessed by aposematic animals (i.e., high contrast at mid-range spatial frequencies) will induce trypophobia. This 
explains why a very large range of stimuli can induce the aversion. Here, we make a similar argument but now view hyperexcitability of individuals with the condition as a necessary (but not sufficient) condition.

Uncomfortable images not only have a spectral profile with excess energy at midrange frequencies (Penacchio \& Wilkins, 2015); they can induce a large haemodynamic response (Haigh et al., 2013; Huang, Cooper, Satana, Kaufman, \& Cao, 2003; Le et al. 2017) and, in general, individuals who are susceptible to discomfort exhibit a larger haemodynamic response than other individuals (reviewed in Wilkins, 2015). Indeed, Wilkins and Hibbard (2014) have proposed that the discomfort is part of a homeostatic mechanism to reduce metabolic load on the visual cortex.

We suggest that trypophobia is driven in part by image statistics and associated hyperexcitability, and in part by disgust/fear, and we acknowledge that high level learning may also play a role. Images of clusters of holes digitally incorporated into human skin make very aversive stimuli particularly when the holes are incorporated in an upright as opposed to an inverted or phase-scrambled face (Furuno, Imaizumi, Maeda, Hibino \& Kayama, 2017). Although images such as these could possess higher contrast than naturally occurring skin lesions, the similarity with skin pathologies may well contribute to the increase in the aversion to such stimuli, given that people with a history of dermatological symptoms show stronger trypophobia (Yamada \& Sasaki, 2017). As with many theories of cognitive phenomena (e.g., attentional 'capture'; Reynolds \& Desimone, 2003) a fuller account of trypophobia will perhaps be one in which both top-down and bottom-up processes are included.

\section{Acknowledgements}

We thank the participants.

\section{Funding}

This research did not receive any specific grant from funding agencies in the public, commercial, or not-for-profit sectors.

\section{References}

American Psychiatric Association. (2013). The Diagnostic and Statistical Manual of Mental Disorders: DSM 5. Washington: American Psychiatric Association.

Ayzenberg, V. Hickey, M.R. \& Lourenco, S.F. (2018). Pupillometry reveals the physiological underpinnings of the aversion to holes. PeerJ 6:e4185; DOI 10.7717/peerj.4185

Cacioppo, J. T., Tassinary, L. G., \& Berntson, G. (Eds.). (2007). Handbook of Psychophysiology (3rd ed.). New York: Cambridge University Press.

Cole, G. G., \& Wilkins, A. J. (2013). Fear of Holes. Psychological Science, 24(10), 1980-1985. doi: 10.1177/0956797613484937

Ekman, P., Levenson, R. W., \& Friesen, W. V. (1983). Autonomic nervous system activity distinguishes among emotions. Science, 221(4616), 1208-1210.

Elgendi, M., Jonkman, M., \& DeBoer, F. (2011). Heart Rate Variability and the Acceleration Plethysmogram Signals Measured at Rest. In A. Fred, J. Filipe, \& H. Gamboa (Eds.), Biomedical Engineering Systems and Technologies (pp. 266-277). Berlin: Springer Berlin Heidelberg.

Gerdes, A.B.M., Uhi, G. Alpers G.W. (2009). Spiders are special: fear and disgust evoked by 
pictures of arthropods. Evolution and human behavior, 30(1), 66-73.

Haigh, S. M., Barningham, L., Berntsen, M., Coutts, L. V., Hobbs, E. S., Irabor, J., . . . Wilkins, A. J. (2013). Discomfort and the cortical haemodynamic response to coloured gratings. Vision Research, 89, 47-53. doi: 10.1016/j.visres.2013.07.003

Hibbard, P. B. \& O'Hare, L. (2015). Uncomfortable images produce non-sparse responses in a model of primary visual cortex. $R$. Soc. open sci. 2: 140535 . http://dx.doi.org/10.1098/rsos.140535

Furuno, M., Imaizumi, S., Maeda, K., Hibino, H., \& Koyama, S. (2017). The influence of background objects on unpleasantness evoked by lotus-seed-pods-on-the-living-body images ("Hasu-colla"). International Journal of Affective Engineering, 16(3), 221-230. doi:10.5057/ijae.IJAE-D-16-00045

Furuno, M., Sakurai, Y., Imaizumi, S., \& Koyama, S. (2018). Face-Inversion Effect on Disgust Evoked by a Cluster of Dots. i-Perception, 9(3), 2041669518784960. doi:10.1177/2041669518784960

Huang, J., Cooper, T. G., Satana, B., Kaufman, D. I., \& Cao, Y. (2003). Visual distortion provoked by a stimulus in migraine associated with hyperneuronal activity. Headache, 43(6), 664-671. doi: 10.1046/j.1526-4610.2003.03110.x

Imaizumi, S., Furuno, M., Hibino, H., \& Koyama, S. (2016). Trypophobia is predicted by disgust sensitivity, empathic traits, and visual discomfort. SpringerPlus, 5(1), 1449. doi: 10.1186/s40064-016-3149-6

Kreibig, S. D. (2010). Autonomic nervous system activity in emotion: A review. Biological Psychology, 84(3), 394-421. doi: 10.1016/j.biopsycho.2010.03.010

Lao, C. K., Che, U. K., Chen, W., Pun, S. H., Mak, P. U., Wan, F., \& Vai, M. I. (2012). Portable Heart Rate Detector Based on Photoplethysmography with Android Programmable Devices for Ubiquitous Health Monitoring System. International Journal of Advances in Telecommunications, Electrotechnics, Signals and Systems, 2(1), 18-26. doi: 10.11601/ijates.v2i1.22

Le, A. T. D., Cole, G. G., \& Wilkins, A. J. (2015). Assessment of trypophobia and an analysis of its visual precipitation. The Quarterly Journal of Experimental Psychology, 68(11), 2304 - 2322. doi: 10.1080/17470218.2015.1013970

Le, A. T. D., Payne, J., Clarke, C., Kelly, M. A., Prudenziati, F., Armsby, E., . . Wilkins, A. J. (2017). Discomfort from urban scenes: Metabolic consequences. Landscape and Urban Planning, 16o, 61-68. doi: 10.1016/j.landurbplan.2016.12.003

Penacchio, O., \& Wilkins, A. J. (2015). Visual discomfort and the spatial distribution of Fourier energy. Vision Research, 108, 1-7. doi: 10.1016/j.visres.2014.12.013

Penacchio, O., Otazu, X., Wilkins, A.J. and Harris, J.M. (2019). Excitation-inhibition balance and sparse coding can jointly account for differences in visual discomfort across different scenes and observers. in final stages of preparation

Pipitone NR, Gallegos B, Walters D. (2017). Physiological responses to trypophobic images and further scale validity of the trypophobia questionnaire. Personality and Individual Differences 108:66-68 DOI 10.1016/j.paid.2016.11.068.

Qualtrics Labs Inc. (2013). Qualtrics (Version 61066). Provo, UT.

Task Force of the European Society of Cardiology and the North American Society of Pacing and Electrophysiology. (1996). Heart rate variability: standards of measurement, physiological interpretation and clinical use. Circulation, 93(5), 1043-1065. doi: 10.1161/o1.cir.93.5.1043

Van Strien, J.W. \& Van der Peijl , M.K. (2018). Enhanced early visual processing in response to snake and trypophobic stimuli. BMC Psycholog 6:21.https://doi.org/10.1186/s40359-0180235-2

Vlok-Barnard, M., \& Stein, D. J. (2017). Trypophobia: an investigation of clinical features. Revista Brasileira de Psiquiatria(AHEAD), o-o. doi: 10.1590/1516-4446-2016-2079 
Wabnegger, A., Schwab, D. \& Schienle, A. (2019). The hole story: an event-related potential study with trypophobic stimuli. Motiv. Emot. 43: 985. https://doi.org/10.1007/s11031-019-09784-8.

Wilkins, A. J. (2015). A physiological basis for visual discomfort: Application in lighting design. Lighting Research and Technology, 1477153515612526. doi: 10.1177/1477153515612526

Wilkins, A., \& Hibbard, P. B. (2014). Discomfort and hypermetabolism. Paper presented at the AISB50, Goldsmith.

Yamada, Y., \& Sasaki, K. (2017). Involuntary protection against dermatosis: a preliminary observation on trypophobia. BMC Research Notes, 10(1), 658. doi:10.1186/s13104-017-2953-6.

Zhao, H., Tanikawa, Y., Gao, F., Onodera, Y., Sassaroli, A., Tanaka, K., \& Yamada, Y. (2002). Maps of optical differential pathlength factor of human adult forehead, somatosensory motor and occipital regions at multi-wavelengths in NIR. Physics in Medicine and Biology, 47(12), 2075. 
Figure Legend

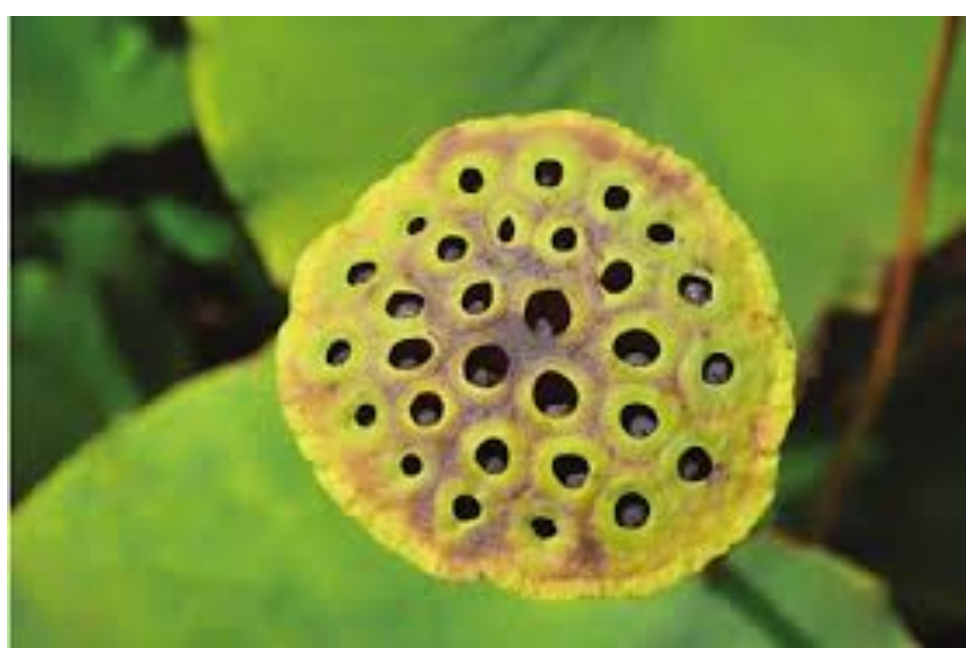

Figure 1. Seed head of the lotus flower. 